\title{
ON THE CLASSICAL STABILITY CONDITIONS FOR A SPINNING SHELL*
}

\author{
BY \\ P. C. RATH AND DAULAT RAM \\ Institute of Armament Technology, Poona, India
}

1. Introduction. Nielsen and Synge [1] for the first time enunciated a set of necessary and sufficient conditions for the stability of a spinning shell. These conditions were further examined by McShane, Kelley and Reno [2] and separately by Murphy [3] and one of the authors [4] under less stringent conditions of motion. McShane and others have applied some of these conditions to plane yawing, motion of bombs and Murphy and Bolz [5] to slow-yawing motion of missiles. Rankin [6] has given similar conditions of stability for rotating uncontrolled rockets. All these only show the importance of Nielsen and Synge's stability conditions and the important role they play in modern exterior ballistics.

In this paper we have derived the classical conditions of Nielsen and Synge from the will-known Routh-Hurwitz theorem. In doing so we have proved that one of the three conditions of Nielsen and Synge is superfluous. Thus there are only two independent conditions which reduce to only one effective condition in the absence of cross-Magnus effects. A mathematical lemma due to Brull and Soler [7] is used to provide logical foundations for certain assumptions of Nielsen and Synge while formulating their stability condition.

2. Certain mathematical preliminaries. We have the following lemmas to start with:

Lemma 1. Given a polynomial $f(Z)$ for which $f(i Z)=\left(b_{0} Z^{n}+b_{1} Z^{n-1}+\cdots+b_{n}\right)+$ $i\left(a_{0} Z^{n}+\cdots+a_{n}\right),\left(a_{0} \neq 0\right)$, where the polynomials $a_{0} Z^{n}+\cdots+a_{n}$ and $b_{0} Z^{n}+\cdots+b_{n}$ are relatively prime $\left(\nabla_{2 n} \neq 0\right)$ then the number of roots of the polynomial $f(Z)$ lying in the right half of the complex plane of $Z$ is given by

$$
k=V\left(1, \nabla_{2}, \nabla_{4}, \cdots \nabla_{2 n}\right)
$$

where

$$
\begin{aligned}
& \nabla_{2 p}=\left|\begin{array}{cccc}
a_{0} & a_{1} & \cdots & a_{2 p-1} \\
b_{0} & b_{1} & \cdots & b_{2 p-1} \\
0 & a_{0} & \cdots & a_{2 p-2} \\
0 & b_{0} & \cdots & b_{2 p-2} \\
\cdots & \cdots & \cdots & \cdots
\end{array}\right| \\
& \left(p=1,2, \cdots, n, a_{m}=b_{m}=0 \text {, if } m>n\right) .
\end{aligned}
$$

If, however, some of the determinants (2.2) vanish, then for each group of successive zeros,

$$
\left(\nabla_{2 h} \neq 0\right), \nabla_{2 h+2}=\cdots=\nabla_{2 h+2 p}=0 \quad\left(\nabla_{2 h+2 p+2} \neq 0\right)
$$

\footnotetext{
${ }^{*}$ Received June 17, 1968; revised version received September 7, 1968.
} 
in the calculation of (2.1) we must set

$$
\operatorname{sign} \nabla_{2 h+2 i}=(-1)^{i(j-1) / 2} \operatorname{sign} \nabla_{2 h} \quad(j=1,2, \cdots, p) .
$$

In the above lemma $V($ ) signifies the number of changes in sign in the determinantal expressions contained in the parentheses.

Lemмa 2. A linear differential equation (with a small parameter $\epsilon$ ) which can be written as

$$
u^{\prime \prime}+f(x, \epsilon) u=0 \quad\left(u^{\prime}=d u / d x\right)
$$

such that

$$
f(x, \epsilon)=g_{0}(x)+G(x, \epsilon) \quad G(x, 0)=0
$$

has the solution

$u(x)=A_{1} w_{1}(x) \exp \left[\sum_{n=1}^{\infty} \epsilon^{n} \int h_{n 1}(x) d x\right]+A_{2} w_{2}(x) \exp \left[\sum_{n=1}^{\infty} \epsilon^{n} \int h_{n 2}(x) d x\right]$

where $A_{i}(i=1,2)$ are arbitrary constants and $w_{i}(x)$ are the linearly independent solutions of

$$
w^{\prime \prime}+g_{0}(x) w=0
$$

and the functions

$$
H_{i}(x, \epsilon)=\sum_{n=1}^{\infty} h_{n i}(x) \epsilon^{n}, \quad(i=1,2)
$$

satisfy a Ricatti equation of the type

$$
H^{\prime}+\left(2 w^{\prime} / w\right) H+H^{2}=-G(x, \epsilon) .
$$

If in any specific case the series in (2.7) converges, then the solution given by (2.7) is exact or else asymptotic for small values of $\epsilon$.

The above two lemmas are known results due to Gantmacher [8] and Brull and Soler [7] respectively.

3. The linearized dynamical equations of the shell and asymptotic stability. Unless otherwise stated the notations we shall use in our present treatment will be strictly according to Nielsen and Synge's paper [1], which we shall refer to as N.S. hereafter. The partially linearized equations of motion of a spinning shell according to N.S. are

$$
\begin{aligned}
\dot{\xi}-i \omega \eta & =\xi X+\eta Y+g \cos \theta, \\
\dot{\eta}-i C^{\prime} \omega_{3} \eta & =\xi X^{\prime}+\eta Y^{\prime} \quad\left(C^{\prime}=C / A\right), \\
\dot{\omega}-u \omega_{2}+v \omega_{1} & =\left(F_{3}+F_{3}^{\prime}\right) / m, \\
\dot{\omega}_{3} & =G_{3} / C .
\end{aligned}
$$

Here overhead dots indicate time derivatives; $\omega, \omega_{3}$ and $\xi, \eta$ are respectively the axial and cross-axial components of the velocity and angular velocity vectors of the shell; the angular coordinates $\theta$ and $\phi$ measure respectively the inclination of the shell axis to the horizontal plane and the rotation of the vertical plane containing the shell axis from a certain fixed vertical plane. $C$ and $A$ are the polar and equatorial moments of inertia of the shell; $g$ the acceleration due to gravity, and $F_{3}, G_{3}$ and $X, Y, X^{\prime}, Y^{\prime}$ are the aerodynamic forces along and normal to the axis of the shell. 
In deriving these equations it is assumed that the initial oscillations of the axis of the shell are such that

(i) the vertical plane through the axis of the shell turns slowly (i.e. $\dot{\phi}$ is small),

(ii) the angle of yaw (i.e. $\xi / \omega$ ) is small.

These are reasonable assumptions which are true in practice. While discussing rapid oscillations of the shell one also treats $\omega$ and $\omega_{3}$ as constants and consequently $X, Y, X^{\prime}, Y^{\prime}$ are also constants; $\cos \theta$ in the first equation of (3.1) is also assumed constant as the projectile is slowly yawing. Effectively, therefore, (3.1) are two linear differential equations with constant coefficients in $\xi$ and $\eta$. For stability of the projectile it is assumed that initial vibrations must damp out; i.e. the cross velocity and cross-spin should not increase but should tend to their equilibrium values. We must therefore discuss the asymptotic stability of the system

$$
\left(\begin{array}{l}
\dot{\xi} \\
\dot{\eta}
\end{array}\right)=\left(\begin{array}{cc}
X & Y+i \omega \\
X^{\prime} & Y^{\prime}+i C^{\prime} \omega_{3}
\end{array}\right)\left(\begin{array}{l}
\xi \\
\eta
\end{array}\right) .
$$

The characteristic equation of the matrix of the system is clearly

$$
f(\lambda) \equiv \lambda^{2}-\left(K_{1}+i K_{2}\right) \lambda+\left(K_{3}+i K_{4}\right)=0
$$

where

$$
\begin{aligned}
& K_{1}=X_{1}+Y_{1}^{\prime}, \\
& K_{2}=C^{\prime} \omega_{3}+X_{2}+Y_{2}^{\prime}, \\
& K_{3}=-C^{\prime} \omega_{3} X_{2}+\omega X_{2}^{\prime}+X_{1} Y_{1}^{\prime}-X_{2} Y_{2}^{\prime}-X_{1}^{\prime} Y_{1}+X_{2}^{\prime} Y_{2}, \\
& K_{4}=C^{\prime} \omega_{3} X_{1}-\omega X_{1}^{\prime}+X_{1} Y_{2}^{\prime}+X_{2} Y_{1}^{\prime}-X_{1}^{\prime} Y_{2}-X_{2}^{\prime} Y_{1} .
\end{aligned}
$$

It may be noted that the complex quantities $X, Y, X^{\prime}, Y^{\prime}$ are of the type

$$
\begin{aligned}
X & =X_{1}+i X_{2}, & X^{\prime} & =X_{1}^{\prime}+i X_{2}^{\prime}, \\
Y & =Y_{1}+i Y_{2}, & Y^{\prime} & =Y_{1}^{\prime}+i Y_{2}^{\prime} .
\end{aligned}
$$

One may further observe that a substitution $\xi+h$ and $\eta+k$ for $\xi$ and $\eta$ in (3.1), where the constants $h$ and $k$ are given by

$$
\begin{aligned}
& h=g \cos \theta\left(Y^{\prime}+i C^{\prime} \omega_{3}\right) /\left\{X Y^{\prime}-X^{\prime} Y+i\left(C^{\prime} \omega_{3} X-\omega X^{\prime}\right)\right\} \\
& k=-g \cos \theta X^{\prime} /\left\{X Y^{\prime}-X^{\prime} Y+i\left(C^{\prime} \omega_{3} X-\omega X^{\prime}\right)\right\},
\end{aligned}
$$

transforms the first two equations of (3.1) to (3.2), written in vector matrix form.

Now the null solutions of (3.2) are asymptotically stable if and only if the roots of (3.3) have negative real parts. The Nielsen and Synge stability condition must therefore follow from conditions under which the roots of the characteristic polynomial $f(\lambda)$ must lie in the left half of the complex plane of $\lambda$. Since the generality of Lemma 1 is not lost when one assumes $a_{0} \neq 0$, as otherwise one may consider the polynomial $i f(Z)$ instead of $f(Z)$, accordingly we modify the characteristic polynomial (3.3) as

$$
i f(Z) \equiv\left(K_{1} \lambda-K_{4}\right)+i\left(-\lambda^{2}+K_{2} \lambda+K_{3}\right)
$$


so that we have

$$
\begin{aligned}
\nabla_{2} & =\left|\begin{array}{rr}
-1 & K_{2} \\
0 & K_{1}
\end{array}\right|=-K_{1}, \\
\nabla_{4} & =\left|\begin{array}{rrrr}
-1 & K_{2} & K_{3} & 0 \\
0 & K_{1} & -K_{4} & 0 \\
0 & -1 & K_{2} & K_{1} \\
0 & 0 & K_{1} & -K_{4}
\end{array}\right| \\
& =K_{1} K_{2} K_{4}+K_{1}^{2} K_{3}-K_{4} \\
& =D, \text { say }
\end{aligned}
$$

and therefore

$$
k=V\left(1,-K_{1}, D\right)
$$

which is the number of changes in sign in the bracketed expressions in (3.11). Our assumption that the real and imaginary parts of (3.8) are prime to each other implies that none of the characteristic roots lie on the imaginary axis. This gives us

$$
D \neq 0 \text {. }
$$

The necessary and sufficient condition of stability now consists in showing that $k=0$ (i.e. none of the characteristic roots lie in the right half of the complex plane). The following are obvious consequences of the Lemma:

$$
\begin{aligned}
& k=2, \quad \text { when } K_{1}>0, \quad D>0 \\
& k=1, \quad \text { when } K_{1} \frac{\geq}{<}, \quad D<0 \\
& k=0, \quad \text { when } K_{1} \leq 0, \quad D>0 .
\end{aligned}
$$

From the above analysis it is clear that the necessary and sufficient conditions of stability are only

$$
\begin{gathered}
K_{1} \leq 0 \\
D \equiv K_{1} K_{2} K_{4}+K_{1}^{2} K_{3}-K_{4}^{2}>0
\end{gathered}
$$

which are the same as (7.13a) and (7.13c) in N.S. but for an additional equality sign in the latter. This equality sign in (3.16) will be there if we stipulate, as in N.S., that $\lambda$ should have a nonpositive real part, but there need not be asymptotic stability. The additional set of conditions of N.S., i.e.

$$
K_{1}^{2}+K_{2}^{2}+4 K_{3} \geq 0
$$

(cf. N.S. (7.13b)), are naturally superfluous. This fact may also be seen from the following elementary considerations. As we know, since the $K$ 's are real and due to (3.16),

$4\left(K_{1} K_{2} K_{4}+K_{1}^{2} K_{3}-K_{4}^{2}\right)+K_{1}^{4}+\left(K_{1} K_{2}-2 K_{4}\right)^{2}=K_{1}^{2}\left(K_{1}^{2}+K_{2}^{2}+4 K_{3}\right)>0$.

Our contention that condition (7.13b) of N.S. is superfluous is established. This is 
perhaps the reason why conclusions following from inequality (3.17) have been earlier found to be contained in (3.16). ${ }^{1}$

4. Brull-Soler solution of the linearized ballistics equations in the absence of crossMagnus effect. Eliminating $\eta$ and $\dot{\eta}$ between the first two equations of the partially linearized equations of N.S., i.e. Eqs. (3.1) of the present paper, we obtain

$$
\ddot{\xi}-\left(K_{1}+i K_{2}\right) \dot{\xi}+\left(K_{3}+i K_{4}\right) \xi+\cdots=0
$$

where

$$
\begin{aligned}
K_{1} & =X_{1}+Y_{1}^{\prime}+\left[\frac{\left(Y_{1} \dot{Y}_{1}+\omega+Y_{2}\right)\left(\dot{\omega}+\dot{Y}_{2}\right)}{Y_{1}^{2}+\left(\omega+Y_{2}\right)^{2}}\right] \\
K_{2} & =X_{2}+Y_{2}^{\prime}+\Omega+\left[\frac{\dot{\omega} Y_{1}+Y_{1} \dot{Y}_{2}-\omega \dot{Y}_{1}-\dot{Y}_{1} Y_{2}}{Y_{1}^{2}+\left(\omega+Y_{2}\right)^{2}}\right] \\
K_{3} & =-\Omega X_{2}+X_{1} Y_{1}^{\prime}-X_{2} Y_{2}^{\prime}+\omega X_{2}^{\prime}-X_{1}^{\prime} Y_{1}+X_{2}^{\prime} Y_{2} \\
& -\left[-\dot{X}_{1}+\left\{\frac{X_{1} Y_{1} \dot{Y}_{1}-X_{2} Y_{1} \dot{Y}_{2}-\dot{\omega} X_{2} Y_{1}+\left(\omega+Y_{2}\right)\left(\dot{\omega} X_{1}+X_{2} \dot{Y}_{1}+X_{1} \dot{Y}_{2}\right)}{Y_{1}^{2}+\left(\omega+Y_{2}\right)^{2}}\right\}\right] \\
K_{4} & =\Omega X_{1}+X_{1} Y_{2}^{\prime}+X_{2} Y_{1}^{\prime}-\omega X_{1}^{\prime}-X_{2}^{\prime} Y_{1}-X_{1}^{\prime} Y_{2} \\
& -\left[-\dot{X}_{2}+\left\{\frac{Y_{1}\left(\dot{\omega} X_{1}+X_{2} \dot{Y}_{1}+X_{1} \dot{Y}_{2}\right)-\left(\omega+Y_{5}\right)\left(-\omega X_{2}+X_{2} \dot{Y}_{1}-X_{2} Y_{2}\right)}{Y_{1}^{2}+\left(\omega+Y_{2}\right)^{2}}\right\}\right]
\end{aligned}
$$

and we put

$$
\Omega=C^{\prime} \omega_{3} .
$$

In (4.1) we have not shown the nonhomogeneous part of the equation as this is not needed for considerations of stability. During the initial oscillations of the shell the terms in the square brackets in (4.2) to (4.5) turn out to be considerably smaller than the remaining terms.

To obtain the transient solutions of (4.1) we make the following substitution:

$$
\xi=u \exp \left\{\frac{1}{2} \int\left(K_{1}+i K_{2}\right) d t\right\}
$$

and (4.1) is then transformed to

$$
\ddot{u}+M u=0
$$

where

$$
M=\left[\frac{1}{2}\left(\dot{K}_{1}+i \dot{K}_{2}\right)\right]-\frac{1}{4}\left(K_{1}^{2}-K_{2}^{2}+2 i K_{1} K_{2}\right)+K_{3}+i K_{4} .
$$

If we neglect the cross-Magnus effects we have

$$
X_{2}=Y_{1}=X_{1}^{\prime}=Y_{2}^{\prime}=0
$$

${ }^{1}$ cf. N. S. p. 218 and [2, p. 621$]$. 
and therefore straight away

$$
\begin{aligned}
& K_{1}=X_{1}+Y_{1}^{\prime}+\left[\left(\dot{\omega}+\dot{Y}_{2}\right) /\left(\omega+Y_{2}\right)\right] \\
& K_{2}=\Omega \\
& K_{3}=X_{1} Y_{1}^{\prime}+\omega X_{2}^{\prime}+X_{2}^{\prime} Y_{2}+\left[\left(\dot{\omega}+\dot{Y}_{2}\right) /\left(\omega+Y_{2}\right)-\dot{X}_{1}\right] \\
& K_{4}=\Omega X_{1} .
\end{aligned}
$$

As before, terms inside square brackets are smaller than the rest of the terms in the above equations. These terms are also completely negligible if we assume with N.S. that during initial oscillations of the shell $\omega$ and $\omega_{3}$ and therefore $X$ 's and $Y$ 's are all constants.

Introducing the dimensionless aerodynamic coefficients referred to the centroid of the shell and the small parameter

$$
\epsilon=\rho a^{3} / m,
$$

we may write, using (8.7) of N.S. that

$$
M=g_{0}+\epsilon g_{1}+\epsilon^{2} g_{2}+\cdots
$$

where

$$
\begin{aligned}
g_{0}= & \Omega^{2} / 4 \\
g_{1}= & {\left[-\frac{\omega}{a} f_{1}^{*}+a \omega k_{t}^{-2}\left\{g_{1}^{\prime *}+\frac{r}{a}\left(g_{2}^{*}+f_{2}^{\prime *}\right)+\frac{r^{2}}{a^{2}} f_{1}^{*}\right\}\right]\left(\frac{i \Omega}{2}\right)-\omega^{2} k_{t}^{-2}\left(f_{2}^{\prime *}+\frac{r}{a} f_{1}^{*}\right) } \\
g_{2}= & -\frac{1}{4}\left[\frac{\omega^{2}}{a^{2}} f_{1}^{* 2}+a^{2} \omega^{2} k_{t}^{-4}\left\{g_{1}^{\prime *}+\frac{r}{a}\left(g_{2}^{*}+f_{2}^{\prime *}+\frac{r^{2}}{a^{2}} f_{1}^{*}\right)\right\}\right] \\
& +\frac{1}{2} \omega^{2} k_{t}^{-2} f_{1}^{*}\left\{g_{1}^{\prime *}+\frac{r}{a}\left(g_{2}^{*}+f_{2}^{\prime *}\right)+\frac{r^{2}}{a^{2}} f_{1}^{*}\right\}-\omega^{2} k_{t}^{-2}\left(f_{2}^{\prime *}+\frac{r}{a} f_{1}^{*}\right)\left(g_{2}^{*}+\frac{r}{a} f_{1}^{*}\right)
\end{aligned}
$$

and

$$
g_{n}=0 \quad(n>2)
$$

where

$$
k_{t}=(A / m)^{1 / 2}
$$

is the transverse radius of gyration of the shell.

Using Lemma 2, we proceed to solve Eq. (4.8) as follows. ${ }^{2}$ For this we consider the two linearly independent solutions of

$$
\ddot{\omega}+\frac{1}{4} \Omega^{2} w=0
$$

in the form

$$
w_{k}=\exp \left\{(-1)^{k} i \Omega t / 2\right\}, \quad(k=1,2)
$$

so that

$$
h_{11}=i g_{1} / \Omega=-h_{12}
$$

${ }^{2}$ As in N. S., we assume $g_{i}{ }^{\prime}$ 's to be constants and effectively (4.8) is an equation with constant coefficient. 


$$
\begin{aligned}
h_{21} & =i \Omega^{-1}\left(g_{2}-g_{1}^{2} / \Omega^{2}\right)=-h_{22} \\
h_{31} & =-2 i \Omega^{-3} g_{1}\left(g_{2}-g_{1}^{2} / \Omega^{2}\right)=-h_{32} \\
h_{41} & =i \Omega^{-5}\left[4 g_{1}^{2}\left(g_{2}-g_{1}^{2} / \Omega^{2}\right)-\Omega^{2}\left(g_{2}-g_{1}^{2} / \Omega^{2}\right)^{2}\right] \\
& =-h_{42}
\end{aligned}
$$

and the solution of (4.8) up to fourth order in $\epsilon$ is therefore

$$
\begin{aligned}
u(t)= & A_{1} \exp \left(i \Omega / 2+\epsilon h_{11}+\epsilon^{2} h_{21}+\epsilon^{3} h_{31}+\epsilon^{4} h_{41}\right) t \\
& +A_{2} \exp \left(-i \Omega / 2+\epsilon h_{12}+\epsilon^{2} h_{22}+\epsilon^{3} h_{32}+\epsilon^{4} h_{42}\right) t
\end{aligned}
$$

using (2.7). For this degenerate case, (4.28) could also have been obtained by solving Eq. (4.8) with constant coefficients and then expanding the solution in powers of $\epsilon$ up to four. Now the final solution in $\xi$ up to this order may be written as

$$
\xi(t)=A(t) \exp \left(\frac{K_{1}}{2}+\phi\right) t+B(t) \exp \left(\frac{K_{1}}{2}-\phi\right) t
$$

where $A(t)$ and $B(t)$ are bounded functions of $t$,

$$
\begin{aligned}
\phi= & -\epsilon g_{12} / \Omega+2 \epsilon^{2} g_{11} g_{12} / \Omega^{3}+2 \epsilon^{3} g_{12}\left(\Omega^{2} g_{2}+g_{12}^{2}-3 g_{11}^{2}\right) / \Omega^{5} \\
& +4 \epsilon^{4} g_{11} g_{12}\left\{5\left(g_{11}^{2}-g_{12}^{2}\right)-3 \Omega^{2} g_{2}\right\} / \Omega^{7} \\
K_{1}= & X_{1}+Y_{1}^{\prime}=-\epsilon a \omega k_{t}^{-2}\left(g_{1}^{\prime *}+\frac{r}{a}\left(g_{2}^{*}+f_{2}^{*}\right)+\frac{k_{t}^{* 2}}{a^{2}} f_{1}^{*}\right)
\end{aligned}
$$

aad $g_{11}, g_{12}$ are the real and imaginary parts of $g_{1}$ and therefore given by

$$
\begin{aligned}
& g_{11}=-\omega^{2} k_{t}^{-2}\left(f_{2}^{\prime *}+\frac{r}{a} f_{1}^{*}\right) \\
& g_{12}=\frac{\Omega}{2}\left[a \omega k_{t}^{-2}\left\{g_{1}^{\prime *}+\frac{r}{a}\left(g_{2}^{*}+f_{2}^{\prime *}\right)+\frac{r^{2}}{a^{2}} f_{1}^{*}\right\}-\frac{\omega}{a} f_{1}^{*}\right] .
\end{aligned}
$$

Noting that

$$
k_{\imath}^{*^{2}}=k_{\imath}^{2}+\gamma^{2}
$$

by the theorem of parallel axis, we may write (4.29) up to first significant term in $\epsilon$ as $\xi(t)=A(t) \exp \left[-\epsilon a \omega k_{t}^{-2}\left\{g_{1}^{\prime *}+\frac{r}{a}\left(g_{2}^{*}+f_{2}^{\prime *}\right)+\frac{r^{2}}{a^{2}} f_{1}^{*}\right\}\right] t+B(t) \exp \left[-\epsilon \frac{\omega}{a} f_{1}^{*}\right] t$.

Now the stability of this solution and therefore of the Brull-Soler solution in general depends on the following conditions:

$$
\begin{gathered}
f_{1}^{*}>0, \\
g_{1}^{\prime *}+\frac{r}{a}\left(g_{2}^{*}+f_{2}^{\prime *}\right)+\frac{r^{2}}{a^{2}} f_{1}^{*}>0 .
\end{gathered}
$$

The second condition of (4.36) is an assumption of N.S., i.e. $Y_{1}^{\prime}<0$ (cf. N.S. (8.14)). The two conditions (4.36) together give rise to the condition (3.15), but (3.16), where the pins 
of the projectile is reflected, is not brought out by the Brull-Soler solution, perhaps because of its approximate nature.

5. Conclusion. The classical conditions of stability of a spinning projectile due to McShane and others [2] were established from an approximate solution of the ballistic equations. ${ }^{3}$ Naturally, therefore, these conditions need not be exhaustive and that they are not is clear from the fact that they do not explain a certain basic phenomenon known as "Magnus instability". It is well known [9] that a projectile with a hemispherical base shape cannot be spun fast enough to stabilize it dynamically, as a very strong negative Magnus force will be acting on the hemispherical base. The coefficients of Magnus moment for such projectiles have been found to be positive, unlike conventional projectiles. The linear theory of stability due to Kelly and McShane should therefore indicate that for a positive Magnus moment coefficient it would not be possible to stabilize a projectile by high spin, which it does not. Perhaps it would be logical if it were possible to derive the stability conditions from the ballistic equations themselves and not from their solution which could at the most be approximate in character.

Acknowledgement. The authors are grateful to the Dean, Institute of Armament Technology, Poona for permission to publish this paper.

\section{RefEREnCES}

[1] K. L. Nielsen and J. L. Synge, On the motion of a spinning shell, Quart. Appl. Math. 4, 201-206 (1946)

[2] E. J. McShane, J. L. Kelly and F. V. Reno, Exterior ballistics, Univ. of Denver Press, Denver, Colorado, 1953

[3] C. H. Murphy, On stability criteria of Kelly-McShane linearized theory of yawing motion, BRL Report 853, Princeton Univ. Press, Princeton, N. J., 1953

[4] P. C. Rath, On the motion of a spinning artillery shell, Proc. Nat. Inst. Sci. India 27A, 233 (1961)

[5] R. E. Bolz, Dynamic stability of a missile in rolling flight, J. Aero. Sci. 19, 395-403 (1952)

[6] R. A. Rankin, The mathematical theory of the motion of rotated and unrotated rockets, Phil. Trans. Roy. Soc. A241, 457-585 (1949)

[7] M. A. Brull and A. I. Soler, A new perturbation technique for differential equations with small parameters, Quart. Appl. Math. 24, 143-151 (1966)

[8] F. R. Gantmacher, The theory of matrices, vol. 2, Chelsea, New York, 1964, p. 248

[9] W. C. Nelson, Selected topics on ballistics, Pergamon Press, New York, 1959, p. 137

${ }^{3} \mathrm{cf} .[2]$, p. 610. 